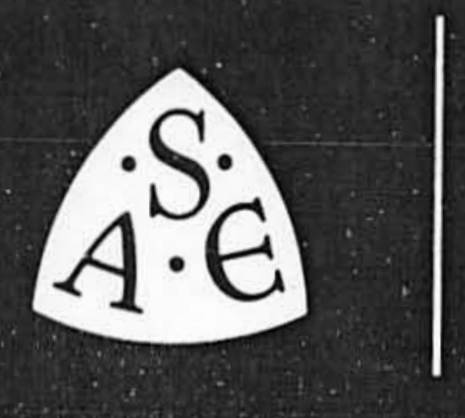

SOCIETY OF AUTOMOTIVE ENGINEERS, INC. Two Pennsylvania Plaza, New York, N.Y. 10001

\title{
Automatic Detection of
}

\section{Intoxicated Drivers}

E. Donald Sussman Philip W. Davis Andrew Warner

Department of 'Trussportation

Transportation Systems Center

Cambridge, Mass. 02142

\section{SOCIETY OF AUTOMOTIVE ENGINEERS}

Automotive Engineering Congress

Detroit, Mich.

January $10-14,1972$
720138 


\section{Automatic Detection of Intoxicated Drivers}

\section{E. Donald Sussman Philip W. Davis \\ Andrew Warner}

Deparment of Transportstion Transportation Systems Center Carbridge, Mass. 02142

As the evidence of the contribution of intoxicated drivers to vehicular fatalities continues to mount, interest has grown in the development of novel countermeasures. One approach now being considered involves the use of a device installed in the automobile which automatically determines if the driver is intoxicated and prevents the driver from operating the vehicle when in- toxication is determined. In this paper such devices are discussed with particular consideration given to the method of determining intoxication, the point in time when the determination is made and their applications. The paper also discusses the Transportation Systems Center's research program directed to dealing with this problem.

\section{INTRODUCTION}

As part of its program to develop methods of reducing the number of alcohol related traffic accidents, the U.S. Department of Transportation (NO'I') is investigating the efficacy of AIcohol Safety Interlock Systems (ASIS). As currently envisoned, these systems perform two functions:

- They automatically determine if the driver is intoxicated;

- They prevent the driver from operating his vehicle if intoxication is determined.

For the purposes of this paper, the term intoxicated refers to the physical and psychological state of an individual with a blood alcohol level (BAL) in excess of $0.10 \%$; the term sober refers to the state of an individual with a BAL of less than 0.048 .

ASIS CLASSIFICATION - ASIS may be classified by considering the method of establishing intoxication, and the point in time at which intoxication is established. 
METHOD OF DETERMINING INTOXICATION

One method of determining intoxication involves estimating the amount of alcohol in the driver's blood through measurement of the driver's breath, tissues, body fluids, or wastes. Measurement of the alcohol in the exhaled alveolar air and estimation of a breath alcohol equivalent ( $B A Q$ ) is a technique currently used by many law enforcement agencies. The technique is attractive because the breath sample is relatively easy to obtain (compared to blood and urine samples) and because a numerical score which is acceptable as evidence in a court of law is obtained. An ASIS based on a breath test would be attractive for these same reasons; however, it would have the following drawbacks:

1. It would be easy to spoof. The driver could simply carry a balloon inflated before he began drinking and apply it to the ASIS whenever he wanted to start his car.

2. It would be specific to impairment caused by alcohol and would be insensitive to the effect of drugs or the synergistic effects of drugs and alcohol.

3. Current breath testing devices use complicated chemical procedures which lend themselves neither to automatic operation nor to low maintenance schedules.

At this time, no ASIS based on chemical tests are available for evaluation. Research into electrochemical sensors for estimating blood alcohol has been undertaken by DOT as well as by private organizations. These sensors are intended for use in cooperative type breath analyzers, and when available will be considered for use in an ASIS.

A second technique involves the measurement of some aspect of the driver's performance or behavior. These methods require a sampling of the driver's performance observed when the driver was known to be sober. A reduction in performance below some criterion is taken to indicate intoxication.

Tasks used to obtain performance indices should meet the following criteria :

1. They should be relatively easy to learn when sober. Extremely complex and difficult tasks might preclude the use of the ASIS on segments of the driving population which could not learn the task or which required such extensive training as to render mass application prohibitively expensive.

2. Task performance should be degraded by the ingestion of alcohol, and the major decrease in performance should occur in the 0.088 to 0.128 BAL range.

3. Task performance should be reliable; that is, it should be approximately the same from day to day at a given BAL for a given individual.

4. The task should use some skill which appears to be related to safe driving. This face validity, while not important to the operation of the ASIS, will influence its acceptability.

Many different tasks have been suggested for sampling performance. The following is a list of those obtained through a review of the scientific literature and contributions solicited from extra governmental agencies.

1. Simple Reaction Response. The driver is required to make a simple motor response as quickly as possible after the occurrance of a stimulus. Only one specific stimulus may occur and only one specific response is required.

2. Complex Reaction Response. The driver is required to correctly choose a response contingent on the occurrance of one particular stimulus from a set of available 
stimuli.

3. Pursuit Tracking. The driver must attempt to match the motion of an externally driven display with a pointer under his or her control, over a period of time.

4. Compensatory Tracking. The driver must attempt to maintain an externally driven display in a null position for some period of time.

5. Divided Attention Task Performance. The driver must perform two tasks simultaneously.

6. Detection of Visual Flicker. The driver must discriminate between flickering and steady visual stimuli.

7. Hand Precision or Steadiness. The driver must perform a task requiring fine manual control.

8. Response Coordination. The driver must perform two different motor responses in close coordination.

A performance type ASIS meeting the previously mentioned criteria would have several advantages.

1. It would be directly sensitive to degradation of the neuro-muscular skills and abilities of the driver induced by the ingestion of alcohol.

2. It could be sensitive to the effects of drugs, the synergistic effects of drugs and alcohol and the synergistic effects of factors such as fatigue and alcohol on driving abilities.

3. Performance type ASIS are not easily spoofed by mechanical analogs.

4. The performance type of ASIS currently available are either electronic or electromechanical and can be made quite durable and tamper resistant.

Performance type ASIS has some disadvantages.

1. The majority of these ASIS require the use of highly practiced tasks to achieve reliability. Widescale use might require the development of extensive training and practice facilities.

2. The majority of these ASIS measure performances with levels which may be idiosyncratic to the driver.

Therefore, a pass/fail score must be set for each driver. This threshold may be difficult to obtain accurately as it would be to the driver's advantage to perform poorly during the tests and set the threshold spuriously low.

3. It is likely that the pass/fail level will be set for each driver by testing him or her when sober and then applying laboratory derived estimates of the effect of intoxication on his score. Due to individual differences in the effect of alcohol on performance, some drivers will be capable of passing the ASIS with BAL's far above the local legal limit; others will fail with BAL's well below this limit.

WHEN INTOXICATION IS DETERMINED - ASIS, which requires the driver to pass a test before starting the vehicle, may be referred to as "hurdle ASIS". ASIS, which sample performance during driving may be referred to as "continuous monitoring ASIS".

The hurdle ASIS are quite simple in operation and may be quite easily interfaced with existing vehicle designs. As the hurdle ASIS must determine intoxication from a short test, some drivers may be able to pass it by marshaling their abilities for a brief period although their performance level over longer periods may be quite low. The hurdle ASIS could allow an individual to start a car immediately after drinking a large quantity of alcohol as performance degradation might not develop until he had been driving for some time. Similarly, hurdle ASIS are not useful in case the 
driver begins drinking after he has

started to drive.

The continuous monitoring ASIS in theory would be sensitive to, the variable of prime interest, driving performance. It could monitor how well the driver was driving and would be sensitive to any factor which degraded driving performance. In order to develop such an ASIS, it would be necessary either to have a metric representing safe driving or to identify some critical aspect of the driving process which was affected by intoxication. In either case, a normal base line would have to be established for the entire population. As no such metric is yet available and as no aspect of the driving task has been demonstrated to be reliably effected by intoxication, performance type continuous monitoring ASIS are presently impractical.

Non-performance continuous monitoring ASIS have also been proposed. These would require the detection of some physiological concommitant of intoxication. No ASIS of this type has been reduced to practical application.

APPLICATIONS - Consideration must be given to the application of the ASIS. One alternative is universal application to all new vehicles. ultimately, every driver would be required to pass the ASIS test each time the vehicle was operated. The cost of such a program would be high. In addition to the expense of the unit itself, the following costs must be considered:

- Installation of the units;

- Inspection of the units:

- Maintenance and repair of the units;

- Training of the drivers in the operation of the units.

It is necessary to balance these

costs against the knowledge that, at present, up to one half the traffic fatalities are attributed to the effects of alcohol. ASIS costs might be reduced considerably by restricting the application of the ASIS to selected segments of the driving population. One possibility is to use the ASIS as an alternative or supportive treatment method for individuals identified as problem drinking drivers. For example, judges might be given the option of restricting problem drinking drivers to operating vehicles with ASIS installed. Licensing agencies might restrict individuals with more than one alcohol related driving offense to vehicles equipped with an ASIS. Other possible applications include inducing drivers statistically identified as high accident risks to install ASIS by providing insurance cost reductions for installation.

CURRENT EFFORTS IN THE DEVELOPMENT OF ASIS At present an investigation of the ASIS concept is being conducted by the Transportation Systems Center (TSC) of DOT with the support of the National Highway Traffic Safety Administration (NHTSA). This effort is divided into three phases.

The first phase, now completed, consisted of a review of the relevant scientific literature and a review of information solicited from extragovernmental organizations. One result of this work* was the identification of a number of promising prototype ASIS which have been developed or are under development. The following is a list of those ASIS units developed by private organizations: PROTOTYPE THEFT PROOF LOCK - Developed by A.S. Dwan L.T.D.; this unit is an ignition lock which requires the driver to carefully set a numbered combination and insert the ignition key with precision. If the *DIscussed in Report No. DOT-TSC-NHTSA71-2. Due to the proprietary material contained in this report, its distribution is restricted to members of the DOT staff. 
driver sets the combination incorrectly, is clumsy in inserting the key, or exceeds the time allowed on the task, he is prevented from starting his vehicle. CRITICAL FLICKER FUSSION TESTER - Developed by Creare Incorporated; this unit requires the operator to discriminate between flickering and steady visual stimuli in order to start his vehicle. The device's ability to determine intoxication is dependent upon a reduction in the critical flicker fusion frequency which accompanies intoxication.

PHYSIOLOGICAL TESTER - Developed by the Delco Electronics Division of General Motors; this unit requires that the driver perform a divided attention task to start his vehicle. The driver must first enter a combination on a "touch tone" type keyboard. If this is done correctly, a five digit number is displayed. The driver must memorize this number which he next enters using the keyboard. At some time during this process a visual stimulus signaling a required brake application will appear on the display. The subject must promptly depress the brake pedal while continuing to enter the number. Failure to perform any of those steps is taken to indicate intoxication. SAFELOCK - Developed by the Nartron Wire Company Incorporated; this device requires a simple manual reaction response to a visual stimulus. A response latency greater than the preset maximum is taken to indicate intoxication.

QUICKEY - Developed by Robert D. Smith; this unit also requires the driver to provide a simple reaction response to visual stimuli, but the method of determining the pass/fail criteria is somewhat different from the Safelock.

For any subject a characteristic response latency for the Quickey is established. This response latency is used to set a passing band such that only a latency which is within ten percent of the characteristic response latency will allow the subject to pass. Responses, either slower or faster than required by the band limits, cause failure. This device determines intoxication through the detection of increased response variability as well as increase response latency. DRUNK DRIVER ELIMINATOR - This unit, developed by the TDL Group of Companies, requires the driver to make closely coordinated, sequenced, manual and pedal responses. Responses too widely separated in time, or inversion of the standard manual then pedal response sequence are considered to indicate intoxication.

The review also revealed a number of measures which might be of use for an ASIS, but had not been reduced to practice. Three ASIS prototypes were developed by TSC to allow testing of these measures. The following are brief descriptions of these TSC developed units. COMPENSATORY TRACKING TESTER - This unit requires the driver to perform a compensatory tracking task. If the driver's absolute error score exceeds a pass/fail threshold, the driver cannot start his vehicle. The threshold is set individually for each driver.

PURSUIT TRACKING TESTER WITH SECONDARY DETECTION TASK - This device requires the driver to perform a pursuit tracking task and simultaneously respond promptly and correctly to a pair of visual stimuli. If the driver's tracking score is above a preset threshold, or if he responds too slowly or incorrectly to the visual stimuli, it is taken as an indication of intoxication. COMPLEX REACTION TESTER - This unit requires the driver to perform a complex reaction task which has both compatible and incompatible stimuli response combinations. The driver is presented with a four stimuli display. The stimuli are composed of four lights arranged as the corners of a rectangle. The display stimuli form two vertical pairs, as the horizontal dimension of the vehicle is much greater than the vertical. The driver must respond to stimuli on the 
upper corners by pressing a button on the same side of the rectangle as the stimulus. This is considered a compatible response. The driver must respond to stimuli on the lower corners of the rectangle by pressing a button on the opposite side of the rectangle from the stimuli. This is considered an incompatible response. Failure to respond promptly and correctly is taken as evidence of intoxication. LABORATORY EVALUATION PROGRAM - The second phase consists of a laboratory evaluation program. This program has been initiated with the cooperation of the Harvard School of Public Health. * The units to be evaluated are described in the previous section.

The results of these tests will be used to determine the following critical factors:

1. How effective are the tests at discriminating between sober and intoxicated individuals? In particular, what is the percentage of false rejections? (False rejections occur when a sober individual fails the ASIS test.) What is the percentage of true rejections? (True rejections occur when an intoxicated individual fails the ASIS test.)

2. How sensitive are the tests to within subject and between subject variability?

3. Will the tests be affected by a problem drinker's (apparent) ability to compensate for alcohol induced changes in performance?

4. What are the training requirements necessary for the potential target drivers?

\#Principal Investigators: Ross McFarland, PH.D., John Dougherty, MD, Guggenheim Center for Aerospace Health and Safcty, Harvard School of Public Health, Boston, Mass.
The laboratory testing has been divided into three segments: pilot rtudies; instrur ment screening tests; final selection and refinement of units.

PILOT STUDIES - The first test segment was used to establish training procedures for the candidate ASIS units, to work out a suitable test schedule, and to generally refine the subject handling and testing procedures.

INSTRUMENT SCREENING TESTS - The second segment, which is underway, is designed to pre-screen the ASIS units for the purpose of determining which units warrant further testing and development. The major goal of this phase is the acquisition of data which will permit the ranking of the test units according to the following two criteria :

- Within Subject Discrimination - The difference between the probability of a given subject passing the tests at low BAQ's (BAQ < .048) and the probability of that subject passing the test of high BAQ's (BAQ>.108).

- Between Subject Discrimination - The difference between the probability of any subject passing at low BAQ's $(B A Q<.048)$ and any other subject passing at a high $\mathrm{BAQ}$ (BAQ > .108). SELECTION AND REFINEMENT OF UNITS - This final segment will be designated to perform more extensive tests on the units that showed promise during the screening tests. In addition, possible improvements in the units will be discussed with the manufacturers.

Approximately 100 subjects are being used in these tests. The majority of them (808) will be male. Two classes of subjects are being used "problem drinkers" obtained with the aid of the Commonwealth of Massachusetts, Registry of Motor Vehicles (508); and "social drinkers" obtained from a pool of volunteers. The subjects are divided into three age groups (under 25, between 25 and 45, and over 45) in order to permit the estimation of the effects of age. 
All of the subjects are paid a base rate plus an incentive bonus based on the number of tests that they pass. This incentive is used to provide motivation similar to that required for actually starting the car.

Subjects are tested in a laboratory environment, but between tests they are permitted to remain in a lounge which is equipped with light reading material, a television set and playing cards. In the testing sessions subjects are administered 195 proof ethyl alcohol mixed with a fruit juice selected by the subject. The dosage is calculated from the body weight of the individual to reach a $B A Q$ of .128 in the "social drinkers" and .168 in the "problem drinkers" after three drinks over a three hour period. FINAL ASIS DEVELOPMENT AND FIELD TESTING After evaluation of the laboratory results, a decision will be made as to the ad- visability and specific direction of future work. If the laboratory tests indicate that one or more of the candidate ASIS units show promise as an effective countermeasure to the drinking driver problem, development and field testing of one or more complete ASIS will be initiated.

The primary purposes for this final phase will be to:

- Optimize the system in terms of physical configuration, interlock techniques, tamperproofing, ignition deactivation times and other operational factors:

- Collect data during routine use of the system by typical target drivers for the purpose of identifying additional problem areas and establishing acceptibility;

- Demonstrate ASIS effectiveness and evaluate total system performance.
This paper is subject to rovislon. Statemiscussion opinions advanced papers or responsibility. not the Society's a however. responsibility not the Society's: however.
the panar raniorms with standards for uniform styling and format. Discussion will be printed with the paper if it is published in SAE Transactions. F or permission to publish this paper in full or in part. contact the SAE Publications Division and the authors. 
8

NOTES 


\section{NOTES}

